\title{
Relações entre tipos de interesse ocupacional e bases psicológicas de comprometimento organizacional
}

\author{
MaurodeOlivera Magglhães- UnivesidadeFeebral da Bahia, Bahia, Brasil
}

\begin{abstract}
Resumo
Os interesses ocupacionais são correlatos importantes de comportamentos e atitudes relacionadas à carreira. Porém, não foram devidamente investigadas as relações entre preferências ocupacionais e atitudes direcionadas à organização empregadora. Esta pesquisa investigou a relação entre interesses ocupacionais e comprometimento organizacional. Foram utilizadas as categorias de interesses descritas por Holland (realista, investigativo, artístico, social, empreendedor e convencional) e o modelo tridimensional do comprometimento organizacional de Meyer e Allen (dimensões afetiva, normativa e instrumental). Uma amostra de 119 homens e 74 mulheres com graduação superior em diversas áreas, idades entre 26 e 57 anos, empregados em organizações da iniciativa privada na região sul do Brasil, participou do estudo. 0 interesse empreendedor foi associado a maior comprometimento de base afetiva. E o comprometimento instrumental foi mais elevado em trabalhadores com interesses artísticos e investigativos. Sugere-se que diferenças de personalidade e valores de carreira relacionados a interesses ocupacionais favorecem atitudes mais instrumentais ou mais afetivas para com a organização empregadora.
\end{abstract}

Palavasdhave Interesses ocupacionais; Comprometimento organizacional; Personalidade.

\section{Relations between types of occupational interests and psychological basis of organizational commitment}

\begin{abstract}
Occupational interests are important correlates of career-related behaviors and attitudes. However, the relationships between occupational preferences and attitudes toward the employer organization were not properly investigated. This research investigated the relation between occupational interests and organizational commitment. The six categories of interest described by Holland (realistic, investigative, artistic, social, enterprising, conventional) and the three-dimensional model of organizational commitment of Meyer and Allen (affective, normative and instrumental dimensions) were utilized. A sample of 119 men and 74 women, graduated in different areas, ages between 26 and 57 years, employed in private organizations of the south of Brazil, participated in the study. The enterprising interest showed association with a greater affective based commitment. The instrumental commitment showed higher scores in workers with artistic and enterprising interests. It is suggested that personality differences and career values related to occupational interests favor instrumental or affective attitudes towards the employer organization.
\end{abstract}

Keguads O ccupational interests; Organizational commitment; Personality.

Relaciones entre tipos de interés ocupacional y bases psicológicas de comprometimiento organizativo

\begin{abstract}
Resumen
Los intereses ocupacionales son correlatos importantes de comportamientos y actitudes relacionados a la carrera. Sin embargo, no fueron debidamente investigadas las relaciones entre preferencias ocupacionales y actitudes direccionadas a la organización empleadora. Esta investigación averiguó la relación entre intereses ocupacionales y comprometimiento organizacional. Fueron utilizadas las categorías de intereses descritas por Holland (realista, investigativo, artístico, social, emprendedor y convencional) y el modelo tridimensional del comprometimiento organizativo de Meyer y Allen (dimensiones afectiva, normativa e instrumental). Una muestra de 119 hombres y 74 mujeres con nivel superior en diversas áreas, edades entre 26 y 57 años, empleados en organizaciones de la iniciativa privada en la región sur de Brasil, participó del estudio. El interés emprendedor fue asociado al mayor comprometimiento de base afectiva. Y el comprometimiento instrumental fue más elevado en trabajadores con intereses artísticos e investigativos. Se sugiere que diferencias de personalidad y valores de carrera relacionados a intereses ocupacionales favorezcan actitudes más instrumentales o más afectivas para con la organización empleadora.

Palabrasdave Intereses ocupacionales; Comprometimiento organizacional; Personalidad.
\end{abstract}

Inúmeros estudos têm sido realizados na tentativa de compreender 0 vínculo indivíduo-organização, considerado um dos fatores estratégicos de sucesso dos projetos empresariais. Esses vínculos e redes de relacionamentos estabelecidos no mundo do trabalho se mostraram os ingredientes de fundo para a emergência de inovações em processos e produtos organizacionais. Entre as linhas de investigação, destaca-se 0 interesse de pesquisadores pelos antecedentes do comprometimento organizacional. As meta-análises de Mathieu e Zajac (1990) e Meyer,
Stanley, Herscovich e Topolnytsky (2002) identificaram cinco categorias de antecedentes do comprometimento organizacional: características pessoais (lócus de controle, autoeficácia relacionada ao trabalho, sexo, entre outras), estados do papel de trabalho (sobrecarga, ambiguidade e conflito), características do emprego, relações com liderança e características da organização. Estudos mais recentes corroboraram e ampliaram esse leque de fatores (Klein, Becker \& Meyer, 2009).

Embora os antecedentes do comprometimento organizacional tenham recebido uma atenção 
consideravel, intluencias ambientais/ contextuais mais do que características pessoais/ disposicionais foram abordadas até 0 momento. No entanto, Erdheim, Wang e Zickar (2006) destacaram o número crescente de pesquisas sobre as fontes disposicionais de atitudes de trabalho. Entre estas, as preferências ou interesses ocupacionais têm se mostrado um fator potencialmente relevante para explicar as características dos vínculos entre 0 trabalhador e a organização. Os interesses ocupacionais já se revelaram correlatos importantes de comportamentos e atitudes relacionadas à carreira, tais como estilo de tomada de decisões (Wright, Reardon, Peterson \& Osborn, 2000), empregabilidade e estratégias de busca de emprego (De Fruyt \& Mervielde, 1999), planejamento e comprometimento com a carreira (Magalhães \& Gomes, 2007). Esta pesquisa investigou a relação entre interesses ocupacionais e as bases psicológicas dos vínculos do indivíduo com a organização. A seguir será apresentado um breve panorama das pesquisas sobre precursores disposicionais de atitudes e comportamentos no trabalho, com ênfase nos vínculos do trabalhador com a organização, e na sequência os construtos enfocados no presente estudo, a saber: 0 modelo de interesses ocupacionais de Holland (1997) e o modelo tridimensional das bases do comprometimento organizacional de Meyer e Allen (1991).

\section{Preursores disposicionais de atitudes e compatamentos no trabalho}

Inúmeras pesquisas encontraram associações entre fatores disposicionais e atitudes $\mathrm{e}$ comportamentos no ambiente de trabalho. Entre os aspectos que se mostraram influenciados por características de personalidade estão o desempenho, satisfação, integração social e comprometimento com a organização (e.g., Joo \& Lim, 2009; Thomas, Whitman \& Viswesvaran, 2010). Portanto, a importância de variáveis disposicionais para 0 vínculo do trabalhador com a organização tem sido destacada por pesquisadores da área.

Essas pesquisas utilizam predominantemente 0 modelo dos cinco grandes fatores de personalidade para avaliar aspectos do indivíduo e o modelo tridimensional do comprometimento organizacional de Meyer e Allen (1991). Os cinco grandes fatores de personalidade são os seguintes: extroversão (intensidade e a quantidade de interações sociais, necessidade de estimulação e capacidade de alegrar-se), abertura à experiência (apreciação da experiência em si mesma, tolerância à ambiguidade e exploração), socialização (dimensão de compaixão vesus antagonismo em pensamentos, sentimentos e ações), realização (organização e persistência) e neuroticismo (ajustamento versusinstabilidade emocional). A sua larga utilização deve-se ao acúmulo de evidências de sua universalidade e aplicabilidade (Nunes, Hutz \& Nunes, 2010).

O modelo teórico do comprometimento organizacional dos canadenses Meyer e Allen (1991) reúne as três vertentes conceituais sobre as bases psicológicas do vínculo entre indivíduo e organização, a saber: afetiva, normativa e instrumental. Em uma descrição sintética, pode-se dizer que os empregados com forte comprometimento afetivo permanecem na organização porque querem (apego emocional, identificação e envolvimento com a organização), os com elevado comprometimento instrumental permanecem porque precisam ou lhes é conveniente (avaliação de custos e benefícios), e aqueles com maior comprometimento normativo permanecem porque se sentem obrigados moralmente a permanecer e contribuir (Meyer \& Allen, 1991).

$O$ estudo de Erdheim, Wang e Zickar (2006) investigou a relação entre os cinco grandes fatores de personalidade e 0 modelo tridimensional do comprometimento organizacional de Meyer e Allen (1991). O traço de extroversão foi preditor das três bases do comprometimento, associado positivamente com a base afetiva e normativa, e negativamente com a instrumental. 0 traço de neuroticismo correlacionou-se positivamente com a base instrumental e mostrou correlações negativas não significativas com as bases afetiva e normativa. 0 traço de realização correlacionou-se positivamente com as bases afetiva e instrumental do vínculo com a organização. Sociabilidade mostrou associação positiva com a base normativa, e a abertura à experiência revelou associação positiva com a base instrumental. Sabendose que à abertura a experiência expressa a necessidade individual por variedade, novidade, complexidade e uma apreciação intrínseca da experiência em si mesma, os autores especularam que pessoas com altos escores nesta dimensão são mais exploratórias e predispostas a buscarem alternativas de emprego em comparação aos baixos escores. Sendo assim, permanecem na organização somente enquanto percebem que isso é vantajoso para suas carreiras e objetivos pessoais, mantendo uma relação instrumental com a instituição. Em relação à sociabilidade, relacionada à convivência social amigável e satisfatória, os autores sugerem que esse traço de personalidade pode elevar a identidade social do trabalhador com seu ambiente de trabalho e, desse modo, aumentar sua necessidade de retribuir à organização por lhe prover esse ambiente social acolhedor, resultando no sentimento de obrigação que caracteriza o vínculo normativo. 


\section{Personalidade, esclhas cupaaionais e compromeimento mo trabalho}

Diferenças de personalidade estão associadas a escolhas ocupacionais, de modo que indivíduos que escolhem a mesma ocupação tendem a possuir traços de personalidade similares (Holland, 1997). Sendo assim, Mount, Barrick, Scullen e Rounds (2005) afirmaram que tanto a taxonomia de interesses de Holland (1997) quanto o modelo dos cinco grandes fatores de personalidade auxiliam na compreensão dos processos motivacionais envolvidos nas preferências dos indivíduos por atividades de trabalho e no grau do seu comprometimento com as mesmas.

No modelo de Holland (1997), os interesses ocupacionais são classificados em seis categorias: realista $(R)$, preferência por atividades mais técnicas e operacionais; investigativo (I), preferência por atividades científicas e intelectuais; artístico (A), preferência por atividades livres, ambíguas e não sistematizadas; social (S), desejo de ensinar e/ ou cuidar de outras pessoas; empreendedor $(E)$, relacionado a atividades de liderança, persuasão e obtenção de ganhos econômicos; e, por fim, a categoria convencional (C), que significa a preferência por atividades estruturadas, com ordens claras e estabelecidas. Os indivíduos expressam preferências de magnitudes diversas associadas a cada categoria e, desse modo, apresentam um perfil de interesses particular. Holland (1997) propôs um modelo hexagonal representativo das correlações entre preferências. No sentido horário, seguindo a ordem RIASEC, cada vértice do hexágono representa a localização de um interesse. Os vértices opostos formam as polaridades realista-social, investigativo-empreendedor e artístico-convencional. Interesses adjacentes estão mais correlacionados, e interesses em vértices opostos são menos correlacionados. Por exemplo, se um indivíduo apresenta seu maior escore em interesse realista $(\mathrm{R})$, seu segundo escore tenderá a ser do tipo convencional (C) ou investigativo (I), e menos provável do tipo social (S).

Diversos estudos das últimas décadas observaram correlações teoricamente consistentes entre os interesses de Holland (1997) e os cinco grandes fatores de personalidade. Costa, McCrae e Holland (1984) encontraram correlações positivas entre interesses investigativos e artísticos e o traço de abertura à experiência, e entre interesses sociais e empreendedores e 0 traço de extroversão. 0 fator socialização diferenciou as preferências ocupacionais de sujeitos mais extrovertidos: aqueles com escores mais altos em socialização preferem ocupações sociais, e os que obtiveram mais baixos preferem ocupações empreendedoras. E o traço realização correlacionou-se com 0 interesse convencional.
Tokar, Vaux e Swanson (1995) investigaram uma amostra de adultos empregados e com interesses de carreira definidos. Os autores consideraram que esses trabalhadores deveriam produzir resultados mais adequados e precisos. De fato, as correlações encontradas se mostraram mais robustas do que em estudos anteriores, corroborando as tendências básicas citadas acima. Estudos mais recentes detalharam as relações encontradas entre interesses e personalidade quanto às facetas dos cinco grandes fatores (e.g., Sullivan \& Hansen, 2004). O estudo brasileiro de Nunes, Hutz e Nunes (2010) encontrou um padrão de correlações com algumas diferenças, onde não ocorreram correlações entre interesse investigativo e 0 traço de abertura e entre interesse convencional e 0 traço de realização. E ainda, nessa amostra brasileira, 0 interesse investigativo correlacionou-se com o traço de realização e as correlações mais elevadas foram encontradas entre 0 interesse artístico e abertura à experiência.

Considerando o que foi dito acima e os achados de Erdheim, Wang e Zickar (2006), pode-se dizer que as correlações encontradas entre interesses sociais e empreendedores, e traços de sociabilidade e extroversão sugerem que trabalhadores com as referidas preferências ocupacionais desenvolvem maior comprometimento de base afetiva. Em comparação, sugerem que trabalhadores com interesses predominantemente artísticos e investigativos apresentem escores mais elevados de comprometimento de base instrumental.

0 profissional de características investigativas tende a voltar-se para recompensas intrínsecas à tarefa, tais como autonomia, aprendizagem e desafio. Em contraste, as inclinações empreendedora e convencional preocupam-se mais com recompensas extrínsecas (salário, benefícios e status) (Amabile, Hill, Hennessey \& Tighe 1994; Magalhães \& Gomes, 2007). Sendo assim, para esses últimos, o reconhecimento interno da organização é mais importante. E trabalhadores de inclinações mais artísticas e investigativas estariam mais interessados no reconhecimento de sua comunidade profissional específica, independentemente da organização empregadora (Fuller, Hester, Barnett, Frey \& Reylea, 2006).

Nesse sentido, a clássica caracterização do comprometimento no trabalho feita por Gouldner (1957) diferenciou trabalhadores cosmopolitas e localistas. Os primeiros teriam baixa lealdade à organização e alto comprometimento com a profissão, pois preocupados com status em grupos de referência das suas áreas específicas. Já os localistas teriam alto comprometimento com a organização, apresentando 
baixo comprometimento com a profissão e competências especializadas. Portanto, de acordo com esta classificação, pode-se sugerir que trabalhadores de inclinações investigativas e artísticas sejam mais cosmopolitas em contraste com aqueles de orientação mais empreendedora e convencional, que seriam mais localistas.

Nesta perspectiva, Fuller e cols. (2006) utilizaram a dicotomia localista-cosmopolita para investigar relações entre comprometimento organizacional, percepção de suporte organizacional e percepção de prestígio externo da organização em 325 funcionários de universidades americanas. Os professores foram enquadrados na definição cosmopolita, e funcionários de staff e administrativos foram enquadrados na categoria localista. Esse enquadramento atendeu a critérios como o grau em que os profissionais estão envolvidos na produção de novos conhecimentos (cosmopolitas) ou na aplicação de conhecimento existente (localistas). Para professores universitários, a produção de conhecimento recebe atenção considerável de processos de avaliação do seu desempenho por agências externas, com consequências quanto ao status e reconhecimento intelectual e financeiro (Tuma \& Grimes, 1981). Por outro lado, pessoal de staff e administradores são avaliados internamente e tendem a apresentar uma orientação mais localista (Keller, 1997; Tuma \& G rimes, 1981).

Em acordo com as expectativas dos autores, os resultados revelaram que a relação entre percepção de suporte organizacional e o comprometimento afetivo foi maior para localistas, e para cosmopolitas foi maior a relação entre comprometimento afetivo e percepção de prestígio externo. Em suma, para funcionários administrativos, o tratamento recebido da organização é mais importante do que o prestígio externo da universidade, e o inverso foi válido para os professores. Professores universitários tendem a preferir atividades investigativas e intelectuais, e funcionários de staff e administrativos tendem a desempenhar atividades convencionais ou empreendedoras em universidades. Sendo assim, esses resultados reforçam a hipótese que profissionais com interesses investigativos apresentem uma orientação mais cosmopolita, com maior inclinação para vínculos instrumentais e menor propensão para vínculos afetivos com suas organizações empregadoras, comparativamente a profissionais de interesses convencionais e empreendedores, onde 0 inverso é esperado.

A distinção entre localistas e cosmopolitas encontra paralelo na contraposição mais recente entre carreira proteana e carreira tradicional. A carreira tradicional é descrita como centrada na organização e nas possibilidades de crescimento que esta oferece, onde sentimentos de lealdade e comprometimento organizacional são predominantes. E a carreira denominada proteana é centrada nas competências pessoais e no aperfeiçoamento contínuo, que permite flexibilidade e adaptabilidade à carreira (Andrade, Kilimnik \& Pardini, 2011). Na pesquisa de Andrade e cols. (2011), as âncoras de carreira (Schein, 1990) empreendedorismo e segurança se mostraram associadas com a carreira tradicional (ou localista), sugerindo uma orientação mais tradicional para a carreira de trabalhadores com interesses ocupacionais empreendedores e convencionais.

\section{Objeivosdbestudo}

A partir dos achados descritos na revisão da literatura, a presente pesquisa teve por objetivo geral investigar relações entre interesses ocupacionais e as três bases psicológicas de vínculo com a organização: afetiva, normativa e instrumental. Os objetivos específicos foram testar hipóteses sugeridas pelas convergências apresentadas nos resultados de estudos anteriores, a saber: (a) escores mais elevados de comprometimento afetivo são encontrados em profissionais com interesses empreendedores e convencionais, em comparação àqueles com interesses investigativos e artísticos; e (b) escores mais elevados de comprometimento instrumental são encontrados em profissionais com interesses investigativos e artísticos, em comparação àqueles com interesses empreendedores e convencionais.

\section{Participantes}

\section{Método}

Uma amostra de 193 profissionais com graduação superior em diversas áreas, idades entre 26 e 57 anos $(\mathrm{M}=42,7 ; \mathrm{DP}=6,7), 119$ homens e 74 mulheres, empregados em organizações da iniciativa privada, com mais de dois anos de vínculo empregatício, responderam a medidas de comprometimento organizacional e interesses ocupacionais.

\section{Instrumentos}

Para medir 0 comprometimento com a organização, foi utilizado o modelo tridimensional das bases do comprometimento organizacional (afetiva, normativa e instrumental) de Meyer e Allen (1991). A escala de comprometimento organizacional foi utilizada na versão brasileira de Medeiros e Enders (1997). Esse instrumento inclui três subescalas referentes às bases afetiva, instrumental e normativa do comprometimento organizacional. Cada subescala é composta de 6 itens. Os itens são frases que descrevem experiências do profissional na sua relação com a organização em que trabalha. 
Para mensurar os interesses ocupacionais, foi utilizada a escala de atividades do Questionário de Busca Auto-dirigida (SeffDireteel Search SD S - Holland, 1997). A versão brasileira do Questionário de Busca Auto-dirigida foi produzida e validada por Primi, Mansão, Muniz e Nunes (2010). O SD S é composto de 4 partes: escala de interesses por atividades, interesses por ocupações, autoestimativas de competências, e autoestimativas de habilidades. A escala de atividades é considerada a mais adequada para a mensuração dos interesses, pois é mais independente de aspectos influenciáveis por treinamento prévio, tais como as habilidades (Yang, Lance \& Hui, 2006). Além disso, por sua representatividade e parcimônia, é recomendada para a coleta de dados em pesquisa. É constituída de seis conjuntos de 11 atividades, correspondentes aos seis tipos de interesse vocacional $(R, I, A, S, E, C)$. No presente estudo, os sujeitos assinalaram sua maior ou menor preferência por esta lista de atividades numa escala Liket de 5 pontos. A soma dos escores em cada conjunto de atividades informa a magnitude de cada categoria de interesse e permite identificar os interesses ocupacionais predominantes no indivíduo.

\section{Procedimentos}

A coleta de dados foi coletiva, realizada no local de trabalho ou estudo dos participantes, com prévio consentimento dos mesmos e das autoridades responsáveis. Em específico, parte dos dados foi coletada em cursos de pós-graduação das áreas de marketing, gestão de pessoas e logística empresarial. A seguir, 0 pesquisador obteve a colaboração de organizações de trabalho, onde os demais participantes preencheram os instrumentos. A pesquisa foi realizada mediante aprovação do Comitê de Ética da instituição de afiliação do pesquisador. Foram atendidas as determinações éticas da resolução 196 do Conselho
Nacional de Saúde (CNS) sobre a pesquisa com seres humanos.

Os sujeitos foram classificados de acordo com 0 interesse ocupacional predominante, tal como avaliado pela escala de atividades do SDS. Foram realizadas ANOVAS para examinar as diferenças de escores nas três bases psicológicas do comprometimento organizacional entre tipos de interesse ocupacional. Este delineamento se repetiu em duas análises. $\mathrm{Na}$ primeira, formaram-se seis agrupamentos ou tipos de interesse ocupacional de acordo com o escore mais elevado (R, I, A, S, E, C). Na segunda, os sujeitos foram agrupados de acordo com os dois interesses adjacentes e com os dois escores mais elevados, sem levar em consideração diferenças de magnitude entre esses dois interesses predominantes (RI, RC, IA, ES, EC, AS). Os perfis foram definidos a partir dos escores brutos, considerando as diferenças intrasujeito, de acordo com as recomendações de Holland (1997). Adverte-se que, na verdade, cada indivíduo possui características de todos os seis tipos em maior ou menor grau, ainda que os atributos de um dado tipo possam predominar. A categorização de um sujeito particular não exige que ele apresente, de modo exclusivo, todos os traços usados para descrever um representante extremo ou ideal de uma inclinação vocacional.

\section{Resultados}

A Tabela 1 apresenta dados de estatística descritiva dos escores de comprometimento organizacional em cada grupo categorizado pelo tipo de interesse ocupacional predominante.

A Tabela 2 apresenta dados de estatística descritiva dos escores de comprometimento organizacional em cada grupo de profissionais categorizado de acordo com os dois interesses adjacentes e com maiores escores.

Tabela 1. Médias e desvios padrão das dimensões de comprometimento organizacional e valores de $\mathrm{N}$ para cada interesse predominante

\begin{tabular}{|c|c|c|c|c|c|c|c|}
\hline \multirow{3}{*}{$\begin{array}{l}\text { Interesse } \\
\text { predominante }\end{array}$} & \multirow{3}{*}{$\mathrm{N}$} & \multicolumn{6}{|c|}{ Bases psicológicas do comprometimento organizacional } \\
\hline & & \multicolumn{2}{|c|}{ Afetiva } & \multicolumn{2}{|c|}{ Normativa } & \multicolumn{2}{|c|}{ Instrumental } \\
\hline & & Média & D esvio padrão & Média & D esvio padrão & Média & D esvio padrão \\
\hline Realista & 13 & 21,85 & 5,18 & 18,31 & 6,58 & 14,85 & 5,32 \\
\hline Investigativo & 35 & 21,74 & 5,45 & 18,23 & 6,00 & 15,57 & 5,30 \\
\hline Artístico & 27 & 20,15 & 5,51 & 16,93 & 5,11 & 15,81 & 4,11 \\
\hline Social & 36 & 21,56 & 4,50 & 16,81 & 5,42 & 15,14 & 4,81 \\
\hline Emprendedor & 76 & 23,51 & 4,63 & 19,76 & 5,65 & 15,30 & 4,77 \\
\hline Convencional & 6 & 21,50 & 6,69 & 14,50 & 5,58 & 14,83 & 6,68 \\
\hline Total & 193 & 22,18 & 5,07 & 18,27 & 5,77 & 15,35 & 4,84 \\
\hline
\end{tabular}


Tabela 2. Médias e desvios padrão das dimensões de comprometimento organizacional e valores de N para cada par adjacente de interesses predominantes

\begin{tabular}{|c|c|c|c|c|c|c|c|}
\hline \multirow{3}{*}{ Interesses Predominantes } & \multirow{3}{*}{$\mathrm{N}$} & \multicolumn{6}{|c|}{ Bases psicológicas do comprometimento organizacional } \\
\hline & & \multicolumn{2}{|c|}{ Afetiva } & \multicolumn{2}{|c|}{ Normativa } & \multicolumn{2}{|c|}{ Instrumental } \\
\hline & & Média & D esvio padrão & Média & D esvio padrão & Média & D esvio padrão \\
\hline Realista-Investigativo & 29 & 22,17 & 5,08 & 18,93 & 5,48 & 14,48 & 4,40 \\
\hline Investigativo-Artístico & 26 & 20,19 & 6,07 & 18,12 & 6,32 & 18,19 & 5,08 \\
\hline Empreendedor-Convencional & 30 & 22,87 & 5,06 & 18,40 & 5,55 & 15,90 & 5,05 \\
\hline Empreendedor-Social & 74 & 23,77 & 4,47 & 18,82 & 5,96 & 14,92 & 4,72 \\
\hline Social-Artístico & 32 & 20,09 & 5,54 & 15,94 & 5,42 & 13,72 & 3,83 \\
\hline Total & 191 & 22,28 & 5,25 & 18,19 & 5,83 & 15,25 & 4,78 \\
\hline
\end{tabular}

As análises de variância, tendo como variável independente os seis agrupamentos definidos pelo interesse mais elevado, indicaram diferenças entre os grupos nos escores de comprometimento organizacional de base afetiva, $F(5,192)=2,73$; $\mathrm{p}<0,05$. Análises post hoc (Tukey) mostraram que indivíduos com interesse empreendedor possuem escores de comprometimento afetivo mais elevado do que indivíduos com interesse artístico.

$\mathrm{O}$ agrupamento dos participantes de acordo com os dois interesses adjacentes no modelo hexagonal e com maiores escores não encontrou indivíduos com 0 perfil RC nesta amostra. Apenas dois participantes foram excluídos desta análise por não apresentarem os dois escores mais elevados em interesses adjacentes no modelo hexagonal. As análises de variância tendo como variável independente os cinco agrupamentos encontrados (RI, IA, ES, EC, SA) indicaram diferenças entre os grupos nos escores de comprometimento organizacional de base afetiva $[F(4,190)=4,27$; $\mathrm{p}<0,01]$ e de base instrumental $[\mathrm{F}(4,190)=3,92$; $\mathrm{p}<0,01]$. Análises post ho (Tukey) revelaram que indivíduos com interesses predominantes dos tipos empreendedor e social (ES) apresentam escores mais elevados na base afetiva do comprometimento em comparação com indivíduos com perfis investigativoartístico (IA) e social-artístico (SA). E em relação à base instrumental, indivíduos com um perfil investigativoartístico (IA) revelaram escores mais elevados em comparação aos perfis artístico-social (AS), empreendedor-social (ES) e realista-investigativo (RI).

\section{Discussão}

Os resultados confirmaram expectativas teóricas. Profissionais com interesses ocupacionais predominantemente empreendedores apresentaram os escores mais elevados em comprometimento organizacional de base afetiva, uma diferença que alcançou significância estatística na comparação com profissionais de interesses predominantemente artísticos. A revisão de literatura apresentou uma série de fatores que podem contribuir para este resultado. Um indivíduo de perfil empreendedor, quando empregado em uma organização específica, tende a estar mais preocupado com o seu status no ambiente interno da organização e com as recompensas extrínsecas do trabalho (remuneração, status) (Amabile \& cols., 1994) e, portanto, mais inclinado a vincular a carreira a uma determinada empresa (Schein, 1990). Desse modo, se aproximam da definição de trabalhadores localistas, uma categoria associada ao maior comprometimento afetivo com a organização e menor com a carreira. Além disso, 0 interesse empreendedor mostrou correlação com a extroversão, um traço de personalidade associado à capacidade de estabelecer conexões sociais positivas no ambiente de trabalho, o que pode ter consequências importantes no comprometimento. Por exemplo, nos estudos de Hurtz e Donovan (2000) e Tziner, Meir e Segal (2002), a extroversão mostrou-se relacionada com avaliações de desempenho positivas por parte de colegas e superiores no local de trabalho. Tziner e cols. (2002) sugeriram que extrovertidos sabem como "vender" suas realizações para seus colegas e superiores, e possuem mais habilidade para deixar uma boa impressão, recebendo melhores avaliações do que introvertidos. Nesse sentido, dadas as habilidades sociais associadas com a extroversão e o interesse empreendedor, compreende-se que este último, entre os grupos estudados, tenha apresentado os escores mais elevados nas bases afetiva e normativa e 0 mais baixo na base instrumental, corroborando os resultados de Erdheim e cols. (2006).

Por outro lado, o interesse artístico se mostrou associado a características de personalidade (abertura a experiência) e a valores de carreira que indicam uma orientação mais cosmopolita nesses profissionais, que seriam propensos a atividades exploratórias de carreira e a mudanças de emprego em busca de objetivos e vantagens pessoais, estabelecendo uma relação menos afetiva com empregadores (Erdheim \& cols., 2006; Holland, 1997). Além disto, no estudo de Nordvik (1996), o interesse artístico foi associado a maior 
preocupação com autonomia, em contraste a preocupações com pertencimento/segurança. E Holland (1997) descreveu esta inclinação de carreira como independente, impulsiva e criativa.

0 agrupamento dos participantes de acordo com os dois interesses adjacentes no modelo hexagonal e com maiores escores não encontrou indivíduos com 0 perfil RC nesta amostra. Este é um perfil pouco comum, tal como evidenciado em pesquisas anteriores com amostras maiores (Magalhães \& Gomes, 2007). As análises de variância tendo como variável independente os cinco agrupamentos encontrados (RI, IA, ES, EC, AS) revelaram que indivíduos de interesses empreendedores e sociais (ES) têm escores mais elevados na base afetiva do comprometimento, em comparação com indivíduos com um perfil investigativo-artístico (IA) e social-artístico (SA). Assim como as inclinações empreendedoras, interesses sociais estão associados com o traço de extroversão, embora com menor intensidade (Tokar \& cols., 1995); e sabendo-se que o comprometimento afetivo se mostrou correlacionado com a extroversão no estudo de Erdheim e cols. (2006), esta combinação de interesses parece fortalecer o comprometimento de base afetiva. É razoável pensar que profissionais com um perfil de interesses empreendedor e social apresentem maior disposição para vincularem-se afetivamente a sua organização de trabalho, pois são capazes de maior interação social e uso de redes de apoio sociopolítico para alcançar suas metas de carreira. No caso de empreendedores, essas metas geralmente envolvem progressão na hierarquia organizacional e ganhos financeiros (Holland, 1997). Em contraste, a introversão característica das inclinações artísticas e investigativas, e a carência de habilidades sociais desses últimos (Holland, 1997), podem ser empecilhos à criação de um vínculo mais afetivo com a organização. Comparados com extrovertidos, introvertidos preferem desenvolver habilidades técnicas e meios de autoexpressão em oposição a competência gerencial e ajudar os demais (Nordvik, 1996).

Por fim, indivíduos com um perfil investigativoartístico (IA) revelaram escores de comprometimento instrumental mais elevado do que indivíduos com perfis artístico-social (AS), empreendedor-social (ES) e realista-investigativo (RI). A combinação de inclinações artísticas e investigativas resultou nos escores mais elevados de comprometimento instrumental. A abertura à experiência é 0 traço marcante de personalidades investigativas e artísticas, e mostrou associação com a base instrumental nos achados de Erdheim e cols. (2006). Além disso, sugere-se que trabalhadores com esses interesses estariam mais voltados para o reconhecimento de sua comunidade profissional, independentemente da organização empregadora, sendo caracterizados como cosmopolitas (Fuller \& colaboradores, 2006). Também tendem a se voltar para recompensas intrínsecas da tarefa, tais como autonomia, aprendizagem e desafio. Em contraste, as inclinações empreendedora e realista preocupam-se mais com recompensas extrínsecas (salário, benefícios e status) (Amabile \& colaboradores, 1994; Magalhães \& Gomes, 2007). Nesse sentido, para esses últimos, 0 reconhecimento interno da organização é mais importante.

Observa-se que a combinação social-artística revelou os menores escores de comprometimento de base afetiva e a associação do interesse artístico com 0 investigativo resultou nos escores mais elevados de comprometimento instrumental. Nesse sentido, a estatística descritiva mostra que trabalhadores com interesse artístico predominante apresentaram os escores mais baixos de comprometimento afetivo e os escores mais elevados de comprometimento instrumental, em comparação aos demais agrupamentos. Estes dados sugerem uma influência importante do interesse artístico nas atitudes afetivas ou instrumentais em relação à organização.

Para a interpretação desses resultados, podem-se fazer associações entre os interesses ocupacionais de Holland (1997), inclinações localistas ou cosmopolitas e o modelo das âncoras de carreira de Schein (1990). A descrição de valores de carreira de pessoas com interesse predominantemente investigativos, artísticos e cosmopolitas se aproxima dos atributos da âncora técnico-funcional, cujos descritores incluem: senso de identidade em torno do conteúdo do trabalho, autonomia, aversão por funções de gerenciamento, preocupação com equidade salarial externa e com 0 reconhecimento de sua comunidade profissional, atração por desafios e aprendizagem na sua especialidade técnica, preferência por benefícios móveis, e maior frequência de transições de carreira. Os valores de carreira comuns em interesses empreendedores e inclinações localistas sugerem uma aproximação com a âncora denominada aptidão administrativa geral, cujos descritores incluem: preocupação com equidade interna e reconhecimento de superiores, preferência por benefícios de estabilidade, tendência a vincular a carreira a uma determinada empresa; e interesses por cargos que signifiquem maiores níveis de hierarquia, responsabilidade, número de subordinados e orçamento.

Portanto, sugere-se que trabalhadores com interesses artísticos e investigativos, assim como os possuidores de âncora técnico-funcional, preocupam-se 
com aspectos intrínsecos do trabalho, com orientação mais cosmopolita de vínculos no trabalho, e traços de introversão. Na direção oposta, profissionais com interesse empreendedor, assim como a âncora administrativa geral, apresentam traços de extroversão, preocupação com aspectos extrínsecos do emprego e orientação mais localista (Amabile \& colaboradores, 1994; Erdheim \& colaboradores, 2006; Keller, 1997; Nordvik, 1996; Schein, 1990). Estas características apontam para uma associação entre interesses investigativos e um comprometimento mais instrumental e menos afetivo com a organização, em comparação a interesses empreendedores, corroborando pesquisas anteriores (Fuller \& colaboradores, 2006; Magalhães \& G omes, 2007).

\section{Considerações finais}

0 conhecimento de diferenças individuais relacionadas a atitudes no ambiente de trabalho é fundamental para oferecer subsídios a lideranças organizacionais e profissionais de recursos humanos, no sentido do atendimento das necessidades diferenciadas de uma força de trabalho heterogênea. Políticas e práticas de gestão e desenvolvimento de pessoas devem ser desenhadas de modo a contemplar essa diversidade de profissionais e carreiras que compõem a vida organizacional.

Entre as limitações deste estudo, é importante salientar que as associações sugeridas entre interesses ocupacionais e as orientações cosmopolitas e localistas precisam ser corroboradas por pesquisas que, além de medidas de interesses ocupacionais e comprometimento organizacional, incluam a avaliação do comprometimento com a carreira. A comparação intrasujeito da magnitude dos vínculos com a carreira $\mathrm{e}$ com a organização torna possível definir perfis de trabalhadores quanto às inclinações localistas e cosmopolitas; e, a seguir, investigar interesses ocupacionais predominantes nesses perfis. Além disso, esta pesquisa não controlou variáveis tais como o setor da economia, cultura e outras características organizacionais que podem ter impacto nos vínculos trabalhador-organização. Sugere-se que amostras maiores e com delimitação de variáveis de ambiente interno e externo da organização possam avançar no entendimento do tema. Por fim, recomendam-se pesquisas brasileiras sobre a relação entre âncoras de carreira e interesses ocupacionais. Essas são sugestões para dar seguimento à investigação das ideias aqui apresentadas.

\section{Referências}

Amabile, T. M., Hill, K. G., Hennessey, B. A., \& Tighe, F. M. (1994). The work preference inventory: assessing intrinsic and extrinsic motivational orientations. Jaumal of Pesanality and Socal Psychdoy, 66, 950-967.

Andrade, G. A., Kilimnik, Z. M., \& Pardini, D. J. (2011). Carreira tradicional versus carreira autodirigida ou proteana: um estudo comparativo sobre a satisfação com a carreira, a profissão e 0 trabalho. Reista deCiênias da Administraçãa 13, 5880.

Costa, P. T., Jr., McCrae, R. R., \& Holland, J. L. (1984). Personality and vocational interests in adulthood. Jaumal of Applied Psydhogy, 69, 390-400.

De Fruyt, F., \& Mervielde, I. (1999). RIASEC types and big five traits as predictors of employment status and nature of employment. Personnd Psychdogy, 52(3), 701-727.

Erdheim, J., Wang, M., \& Zickar, M. (2006). Big five personality constructs and organizational commitment. Pessanalityand Indvidual Differences, 41, 959-970.

Fuller, J. B., Hester, K., Barnett, T., Frey, L., \& Relyea, C. (2006). Perceived organizational support and perceived external prestige: predicting organizational attachment for university faculty, staff, and administrators. The Joumal of Social Psychdogy, 146(3), 327-347.

Gouldner, A. W. (1957). Cosmopolitans and locals: toward an analysis of latent social roles. AdministrativeScienceQuartely, 2, 281-306.

Holland, J. L. (1997). Makingvocational dhices a theery of vocational pessonalities and work enironments O dessa, FL.: PAR.

Hurtz, G. M., \& D onovan, J. J. (2000). Personality and job performance: the five revisited. Jamal of AppliedPsychlogy, 85(6), 869-879.

Joo, B., \& Lim, T. (2009). The effects of organizational learning culture, perceived job complexity, and proactive personality on organizational commitment and intrinsic motivation. Jaumal of Leadeship\& Organizational Studies, 16(1), 48-60.

Keller, R. T. (1997). Job involvement and organizational commitment as longitudinal predictors of job performance: a study of scientists and engineers. Jaumal of Applied Psydhdogy, 82, 539545 . 
Klein, H. J., Becker, T. E., \& Meyer, J. P. (2009). Commitment in arganizations acumulated wisdom and newdiretions Nova Iorque: Taylor \& Francis.

Magalhães, M. O., \& Gomes, W. B. (2007). Personalidades vocacionais e processos de carreira na vida adulta. Psicdogja emEstudo, 12(1), 95-103.

Mathieu, J., \& Zajac, D. (1990). A review and metaanalysis of the antecedents, correlates and consequences of organizational commitment. Psychdogical Bullein, 108, 171-94.

Medeiros, C., \& Enders, W. (1997). Validação de modelo de conceituação de três componentes do comprometimento organizacional de Meyer e Allen (pp. 134). Amais do $21^{\circ}$ Encontroda ANPAD, Rio de Janeiro, Rio das Pedras.

Meyer, J. P., \& Allen, N. J. (1991). A three-component conceptualization of organizational commitment. Human ResarceManagement Review, 1, 61-68.

Meyer, J. P., Stanley, D. J., Herscovich, L., \& Topolnytsky, L. (2002). Affective, continuance, and normative commitment: a meta-analysis of antecedents, correlates and consequences. Jaumal ofV oational Behavior, 61, 20-52.

Mount, M. K., Barrick, M. R., Scullen, S. M., \& Rounds, J. (2005). Higher-order dimensions of the big five personality traits and the big six vocational interest types. Pessannd Psychdogy, 58(2), 447-478.

Nordvik, H. (1996). Relationships between Holland's vocational typology, Schein's career anchors and Myers-Briggs' types. Jaumal of Ocuupational and Organizational Psydhdogy, 69, 263-275.

Nunes, C. H. S. S., Hutz, C. S., \& Nunes, M. F. O. (2010). Bateia Fatorial de Pescnalidade (BFP) manual ténica São Paulo: Casa do Psicólogo.

Primi, R., Mansão, C. M., Muniz, M., \& Nunes, M. F. O . (2010). SDS - QuestionániodeBusca AutoDingida: manual ténico da vesãobrasilèra. São Paulo: Casa do Psicólogo.
Schein, E. (1990). Career andhars San Diego, CA: Pfeiffer \& Company.

Sullivan, B. A., \& Hansen, J. C. (2004). Mapping associations between interests and personality. Jaumal of CaunsdingPsydhogy, 51(3), 287-298.

Tokar, D. M., Vaux, A., \& Swanson, J. L. (1995). Dimensions relating Holland's typology and the five-factor model. Jaumal of Career Assesment, 3, 5774.

Thomas, J. P., Whitman, D. S., \& Viswesvaran, C. (2010). Employee proactivity in organizations: a comparative meta-analysis of emergent proactive constructs. Jaimal of Occupational and Organizational Psychdogy, 83, 275-300.

Tuma, N. B., \& Grimes, A. J. (1981). A comparison of models of role orientation of professionals in research-oriented university. Administrative Saence Quartely, 26, 187-206.

Tziner, A., Meir, E. I., \& Segal, H. (2002). Occupational congruence and personal taskrelated attributes: how do they relate to work performance? Jaumal of Career Assesment, 10(4), 401-413.

Wright, L. K., Reardon, R. C., Peterson,, G. W. \& O sborn (2000). The relationship among constructs in the Career Thoughts Inventory and the SelfDirected-Search. Jaumal of Carer Assesment, 8(2), 105-117.

Yang, W., Lance, C. E., \& Hui, H. C. (2006). Psychometric properties of the Chinese SelfDirected Search (1994 edition). Jaumal of Vocational Behaviar, 68, 560-57.

Sobre o autor:

Mauro de Oliveira Magalhães é Psicólogo (PUCRS, 1989), Mestre em Psicologia (UFRGS, 1995) e D outor em Psicologia (UFRGS, 2005). Vice-presidente da Associação Brasileira de O rientação Profissional (ABOP) (1995-1997; 2005-2007). Editor da Revista Alethia (2002-2008) e da Revista Psicologia O rganizações e Trabalho (2011-atual). Professor na Pós-graduação em Psicologia da Universidade Federal da Bahia. Pesquisador dedicado à psicologia da orientação e desenvolvimento de carreiras.

Contato com o autor:

Rua Tenente Pires Ferreira 308, Bloco A, Apto. 201 - CEP 40130-160. Salvador, Bahia.

Email: mauro.m@terra.com.br 